\title{
Inversion of Quantum Jumps in Quantum Optical Systems under Continuous Observation
}

\author{
H. Mabuchi \\ Norman Bridge Laboratory of Physics 12-33, California Institute of Technology, Pasadena, California 91125 \\ P. Zoller \\ Institut für Theoretische Physik, Universität Innsbruck, Technikerstrasse 25, A-6020 Innsbruck, Austria
}

(Received 11 January 1996)

\begin{abstract}
We formulate conditions for invertibility of quantum jumps in systems that decay by emission of quanta into a continuously monitored reservoir. We propose proof-of-principle experiments using techniques from cavity quantum electrodynamics and ion trapping, and briefly discuss the relevance of such methods for error correction in quantum computation. [S0031-9007(96)00057-9]
\end{abstract}

PACS numbers: 42.50.Lc, 42.50.-p, 89.70.+c

Many current investigations of fundamental quantum phenomena would benefit greatly from the implementation of methods to stabilize quantum states against noise and dissipation [1]. For example, the realizability of quantum computers [2] seems to depend critically on development of robust techniques for preserving the coherence of quantum memory elements. In this Letter we shall describe a scheme for inversion of quantum jumps which, under ideal experimental conditions, makes possible the complete preservation of quantum coherences within a subspace of initial states for specially constructed systems in quantum optics. In the context of quantum computation, our scheme provides a means for dissipation-free storage of quantum bits (qubits).

Decoherence and decay of a quantum optical system may be viewed as the result of weak coupling between the system of interest and a reservoir of electromagnetic field modes whose correlation time is much shorter than the time scale set by system dynamics [1,3]. Under the assumption of vanishing correlation time (Markov approximation), one typically traces over reservoir states in the global equations of motion to derive a master equation that describes evolution of the reduced density operator $\hat{\rho}$ for the system alone. The master equation for $j=\{1, \ldots, d\}$ decay channels is $(\hbar=1)$

$$
\begin{aligned}
\frac{d}{d t} \hat{\rho}= & -i[\hat{H}, \hat{\rho}] \\
& +\sum_{j=1}^{d}\left(\hat{c}_{j} \hat{\rho} \hat{c}_{j}^{\dagger}-\frac{1}{2} \hat{c}_{j}^{\dagger} \hat{c}_{j} \hat{\rho}-\hat{\rho} \frac{1}{2} \hat{c}_{j}^{\dagger} \hat{c}_{j}\right),
\end{aligned}
$$

where $\hat{H}$ is the system Hamiltonian and $\left\{\hat{c}_{j}\right\}$ are the system operators that appear in the system-reservoir coupling. Such a master equation will generally map pure states of the system into statistical mixtures, reflecting the decoherence which results from loss of information into the unobserved reservoir modes.

Indeed, by tracing over the reservoir state to derive (1), one implicitly and essentially assumes that no measurements are ever performed on the reservoir. Much recent work in quantum optics has investigated the a posteri- ori dynamics obtained in contrasting situations where all $j=\{1, \ldots, d\}$ output channels are continuously monitored by ideal photodetectors [4]. For a given count trajectory $j_{1}, t_{1}, \ldots, j_{n}, t_{n}$, the backaction corresponding to observation of count $j_{r}$ at time $t_{r}$ leads to a collapse of the system wave function (quantum jump) described by

$$
\tilde{\psi}_{c}\left(t_{r}+d t\right)=\hat{c}_{j_{r}} \tilde{\psi}_{c}\left(t_{r}\right) .
$$

Here $\hat{c}_{j}$ denotes the system jump operator corresponding to counts in channel $j$, while $\hat{H}_{\text {eff }}=\hat{H}-i \frac{1}{2} \sum_{j} \hat{c}_{j}^{\dagger} \hat{c}_{j}$ is an effective non-Hermitian Hamiltonian. Between counts, the system wave function obeys a Schrödinger equation

$$
\tilde{\psi}_{c}(t)=e^{-i \hat{H}_{\mathrm{eff}}\left(t-t_{r}\right)} \tilde{\psi}_{c}\left(t_{r}\right) .
$$

This quantum-jump picture of dissipative dynamics underlies the recently developed "quantum trajectories" method for Monte Carlo integration of quantum optical master equations [5]. Starting from a known initial (pure) state, count trajectories $j_{1}, t_{1}, \ldots, j_{n}, t_{n}$ may be generated by taking the probability density for a jump to occur at time $t$ to be $\left\|\hat{c}_{j} \psi_{c}(t)\right\|^{2}$. Using the a posteriori evolution rules described above, the system wave function at time $t$ is then given by the normalized state vector $\psi_{c}(t)=$ $\tilde{\psi}_{c}(t) /\left\|\tilde{\psi}_{c}(t)\right\|$. For a physical system in which the count trajectories are not actually detected and recorded, one can average over Monte Carlo wave functions to recover a system density operator $\hat{\rho}=\left\langle\mid \psi_{c}\right\rangle\left\langle\psi_{c} \mid\right\rangle$ which obeys the quantum master equation (1). However, for a laboratory setup which actually incorporates complete and continuous photodetection, the individual trajectories and a posteriori dynamics may be interpreted (with some caution) as reflecting the "real" dynamics of single quantum realizations. This principle motivates our scheme for the preservation of quantum coherence in dissipative systems - if discrete quantum jumps constitute the entire noncoherent component of Markovian dynamics, one can indeed hope to suppress decoherence by performing appropriate operations to invert the operation $\hat{c}_{j_{r}}$ every time a count of type $j_{r}$ is recorded. In general, however, a quantum jump 
(2) will destroy superpositions so that information is irreversibly lost. Hence $\hat{c}_{j}$ will not necessarily be invertible on the entire system Hilbert space $\mathcal{H}$.

Let us therefore formulate conditions under which a quantum jump (2) can be inverted for a system initial state $\tilde{\psi}_{c}(t)$ which is known to lie within a certain subspace $\mathcal{H}_{s} \subset \mathcal{H}$ of the system Hilbert space. We are particularly interested in the case where a detected quantum jump $\tilde{\psi}_{c} \in \mathcal{H}_{s} \rightarrow \tilde{\psi}_{c}^{\prime}=\hat{c}_{j} \tilde{\psi}_{c} \in \mathcal{H}_{s}^{(j)}$ can be inverted using feedback [6] described by a unitary time evolution operator $\hat{U}_{j}$, so that $\tilde{\psi}_{c}\left(t_{r}+d t\right)=\hat{U}_{j} \hat{c}_{j_{r}} \tilde{\psi}_{c}\left(t_{r}\right) \propto \tilde{\psi}_{c}\left(t_{r}\right)$. Thus, as a first condition (A), we require

$$
\hat{c}_{j}=\left.k_{j} \hat{U}_{j}^{-1}\right|_{\mathcal{H}_{s} \rightarrow \mathcal{H}_{s}^{(j)}} \quad\left(k_{j} \in C\right) ;
$$

together with the inverse relation $\hat{c}_{j}^{\dagger}=\left.k_{j}^{*} \hat{U}_{j}\right|_{\mathcal{H}_{s}^{(j)} \rightarrow \mathcal{H}_{s}}$, i.e., for the mapping $\hat{c}_{j}: \mathcal{H}_{s} \rightarrow \mathcal{H}_{s}^{(j)}$ there exists a unitary extension $\hat{U}_{j}$ to the whole Hilbert space [7] which can be generated by an appropriate feedback Hamiltonian. The feedback is assumed to be instantaneous on the time scale of the system dynamics. Equation (4) implies $\hat{c}_{j}^{\dagger} \hat{c}_{j}=\left.\left|k_{j}\right|^{2} \mathbb{1}\right|_{\mathcal{H}_{s} \rightarrow \mathcal{H}_{s} \text {. If we add the requirement (B) that }}$ the system Hamiltonian $\hat{H}$ leaves the subspace of interest $\mathcal{H}_{s}$ invariant, the system dynamics between two quantum jumps is governed by

$$
\begin{aligned}
\tilde{\psi}_{c}(t) & =e^{-i \hat{H}_{\text {eff }} t} \tilde{\psi} \\
& =e^{-1 / 2 \sum_{j}\left|k_{j}\right|^{2} t} e^{-i \hat{H} t} \tilde{\psi} \quad\left[\tilde{\psi}_{c}(t) \in \mathcal{H}_{s}\right]
\end{aligned}
$$

so that the damping terms factor out and thus do not distort the system dynamics between jumps. Furthermore, if each decay is detected and is followed by a feedback $\hat{U}_{j}$ to "undo" the effect of the quantum jump, we have essentially eliminated the effects of decoherence on system states in the subspace $\mathcal{H}_{s}$ :

$$
\begin{aligned}
\psi_{c}(t) & =e^{-i \hat{H}_{\text {eff }}\left(t-t_{n}\right)} \hat{U}_{j_{n}} \hat{c}_{j_{n}} \cdots \hat{U}_{j_{1}} \hat{c}_{j_{1}} e^{-i \hat{H}_{\text {eff }} t_{1}} \psi /\|\cdots\| \\
& =e^{-i \hat{H} t} \psi,
\end{aligned}
$$

where $\|\cdots\|$ denotes normalization of the state.

For the derivation of Eq. (5) to be valid, it is essential that the system dynamics conform to the model of a quantum Markov process [1]. The underlying physical assumption is a separation of time scales where the correlation time $\tau_{c}$ of the environment is much shorter than all time scales characterizing the system evolution, including, in particular, the system decay time [3]. This separation admits the treatment of system dynamics with "coarse-grained" time resolution, and it is only on coarse timescales $\left(\gg \tau_{c}\right)$ that the system wave function appears to evolve according to a non-Hermitian Hamiltonian (3) with stochastic, "instantaneous" quantum jumps [Eq. (2)]. Likewise, it is only on coarse time scales that coherence can be preserved in the system according to Eq. (5), while the state of the environment will not (and need not) be restored at all in the present scheme. We wish to further stress that quantum optical systems are known to realize quantum Markovian models to an excellent approximation, so we fully expect our conclusions drawn from this assumption to be directly applicable to realistic experimental systems.

Significantly, the type of jump-inversion procedure described above seems to be realizable with familiar experimental techniques in several systems of current interest in quantum optics. Our first example utilizes recent ideas from the field of cavity quantum electrodynamics (CQED) [8]. Consider the apparatus shown in Fig. 1, in which the output modes of two identical single-sided Fabry-Pérot resonators are mixed by a 50/50 beam splitter before impinging upon photon-counting detectors. We assume that the high-reflector (HR) mirror of each resonator is perfect, and that the output couplers (OC) have no scattering or absorption losses but have some small transmissivity $t>0$. The beam splitter is likewise assumed to be lossless, and we treat the photodetectors as having unit quantum efficiency. Note that we are not invoking any sort of Zeno effect, so that the time resolution of the detectors is taken as being very short compared to the cavity decay times but long compared to the optical time scale $\sim 1 / \omega_{0}\left(\omega_{0}\right.$ being the optical frequency of the resonator modes). We assume a separation of time scales in which all operations described below can be performed in a time much less than the cavity decay times, which we assume to be equal.

Let $\hat{a}$ and $\hat{b}$ be the annihilation operators for the optical modes of cavities $\mathbf{a}$ and $\mathbf{b}$, respectively. The master equation for the resonator modes may be written

$$
\hat{\rho}=-i\left(\hat{H}_{\mathrm{eff}} \hat{\rho}-\hat{\rho} \hat{H}_{\mathrm{eff}}^{\dagger}\right)+\Gamma\left(\hat{a} \hat{\rho} \hat{a}^{\dagger}+\hat{b} \hat{\rho} \hat{b}^{\dagger}\right),
$$

with $\hat{H}_{\text {eff }}=\left(\omega_{0}-i \frac{1}{2} \Gamma\right)\left(\hat{a}^{\dagger} \hat{a}+\hat{b}^{\dagger} \hat{b}\right)$ the effective Hamiltonian. We identify $\hat{a}$ and $\hat{b}$ as the jump operators

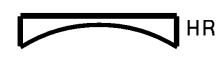

a

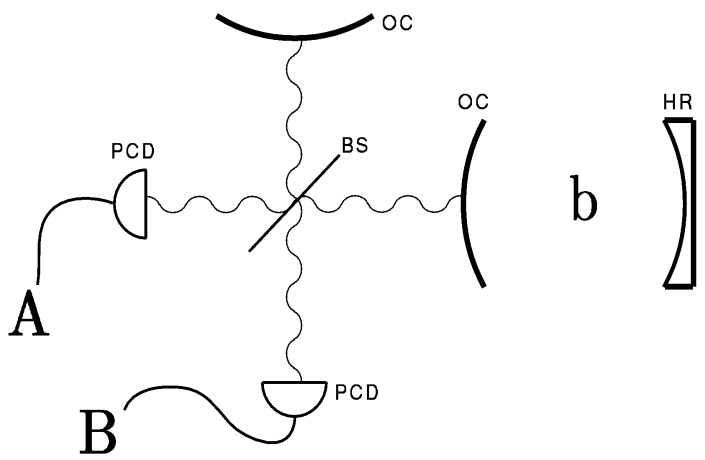

FIG. 1. Schematic of a cavity-QED gedanken experiment. Components are labeled (see text) HR, high-reflector mirrors; OC, output-coupler mirrors; BS, 50/50 beam splitter; and PCD, photon-counting detectors. 
associated with the detection of dissipative events in cavities $\mathbf{a}$ and $\mathbf{b}$. In order to account for the mixed-output measurement scheme of Fig. 1, we must make a basis transformation by defining

$$
\hat{A}=\frac{1}{\sqrt{2}}(\hat{a}+\hat{b}), \quad \hat{B}=\frac{1}{\sqrt{2}}(\hat{a}-\hat{b}),
$$

which we interpret as jump operators corresponding to the registration of photons by detectors $\mathbf{A}$ and $\mathbf{B}$. In terms of the new jump operators, Eq. (6) becomes

$$
\hat{\rho}=-i\left(\hat{H}_{\mathrm{eff}} \hat{\rho}-\hat{\rho} \hat{H}_{\mathrm{eff}}^{\dagger}\right)+\Gamma\left(\hat{A} \hat{\rho} \hat{A}^{\dagger}+\hat{B} \hat{\rho} \hat{B}^{\dagger}\right),
$$

where $\hat{H}_{\text {eff }}=\left(\omega_{0}-i \frac{1}{2} \Gamma\right)\left(\hat{A}^{\dagger} \hat{A}+\hat{B}^{\dagger} \hat{B}\right)$ remains invariant under the change of basis.

In this example we consider stabilization of the subspace spanned by the Fock states $|0\rangle_{L} \equiv\left|2_{a} 0_{b}\right\rangle$ and $|1\rangle_{L} \equiv\left|0_{a} 2_{b}\right\rangle$, representing a logical zero and one, respectively. Let the initial state of the two-cavity system be given by

$$
|\psi\rangle=c_{0}\left|2_{a} 0_{b}\right\rangle+c_{1}\left|0_{a} 2_{b}\right\rangle \equiv c_{0}|0\rangle_{L}+c_{1}|1\rangle_{L} .
$$

We first note that states of this form are stationary under the time evolution (3), since $\left|2_{a} 0_{b}\right\rangle$ and $\left|0_{a} 2_{b}\right\rangle$ are degenerate eigenstates of $\hat{H}_{\text {eff }}$. Therefore the superposition (9) remains unchanged during periods of time in which no photons are detected. When photodetection events do occur, the postjump state $\left|\psi_{c}\right\rangle$ will be either

$$
\begin{aligned}
& \hat{A}|\psi\rangle=c_{0}\left|1_{a} 0_{b}\right\rangle+c_{1}\left|0_{a} 1_{b}\right\rangle, \\
& \hat{B}|\psi\rangle=c_{0}\left|1_{a} 0_{b}\right\rangle-c_{1}\left|0_{a} 1_{b}\right\rangle .
\end{aligned}
$$

As both coefficients $\left(c_{0}, c_{1}\right)$ survive in either case, and remain attached to orthogonal state vectors, the original state $|\psi\rangle$ may, in principle, be fully restored by the application of the appropriate feedback operator $\hat{U}_{A}$ or $\hat{U}_{B}$. Note that one knows which of these to apply based upon which detector registered the photon. The inverse jump operators correspond to the doubling of the photon number in both resonators $(0 \rightarrow 0,1 \rightarrow 2)$, with or without a phase change of $\pi$ in resonator $\mathbf{b}$. Since we must employ only coherent processes for the doubling operation, the states of resonators $\mathbf{a}$ and $\mathbf{b}$ may be independently manipulated without compromising the entanglement between them. This allows us to consider the simplified task of "independently" doubling the photon number in each resonator.

We now proceed to give an explicit example of a process to achieve this photon-number doubling. Our proposal employs adiabatic state-mapping techniques described in [9], by which one can "swap" the state of a resonator field with the internal Zeeman state of an atom. Consider an atom having an angular momentum $J \rightarrow J-1$ transition $(J>1)$ with frequency $\omega_{0}$, prepared in the $\left|g_{m_{J}=-J}\right\rangle$ ground state as depicted in Fig. 2. If we wish to invert an $\hat{A}$-type jump, the combined state of the atom plus resonator fields will initially be

$$
|\Psi\rangle=\left|g_{-J}\right\rangle \hat{A}|\psi\rangle=\left|g_{-J}\right\rangle\left(c_{0}\left|1_{a} 0_{b}\right\rangle+c_{1}\left|0_{a} 1_{b}\right\rangle\right) .
$$

After performing adiabatic state mapping [assuming the resonator mode has $\sigma_{+}$polarization, see Fig. 2(a)],

$$
|\Psi\rangle \rightarrow\left(c_{0}\left|g_{-J+1}\right\rangle\left|0_{b}\right\rangle+c_{1}\left|g_{-J}\right\rangle\left|1_{b}\right\rangle\right)\left|0_{a}\right\rangle .
$$

We can now effect the photon-number doubling for resonator a by applying a Raman $\pi$ pulse to the atom, with $\pi$ - and $\sigma_{-}$-polarized lasers having frequency $\omega_{0}-$ $\delta$. The detuning $\delta$ should be chosen large enough to eliminate any possibility of populating the excited atomic state. After the $\pi$ pulse, we have [Fig. 2(b)]

$$
|\Psi\rangle \rightarrow\left(c_{0}\left|g_{-J+2}\right\rangle\left|0_{b}\right\rangle+c_{1}\left|g_{-J}\right\rangle\left|1_{b}\right\rangle\right)\left|0_{a}\right\rangle .
$$

Note that polarization selection rules prevent the $|-J\rangle$ atomic state from coupling to the specified Raman fields. With a final "reverse" application of the state-mapping procedure [Fig. 2(c)], the total state of the system becomes

$$
|\Psi\rangle \rightarrow\left|g_{-J}\right\rangle\left(c_{0}\left|2_{a} 0_{b}\right\rangle+c_{1}\left|0_{a} 1_{b}\right\rangle\right) .
$$

Thus the photon-number doubling has been accomplished for the first resonator. An analogous procedure for resonator $\mathbf{b}$ completes the process, with the sequence of intermediate states given by

$$
\begin{aligned}
\left|g_{-J}\right\rangle\left(c_{0}\left|2_{a} 0_{b}\right\rangle+c_{1}\left|0_{a} 1_{b}\right\rangle\right) & \rightarrow\left(c_{0}\left|g_{-J}\right\rangle\left|2_{a}\right\rangle+c_{1}\left|g_{-J+1}\right\rangle\left|0_{a}\right\rangle\right)\left|0_{b}\right\rangle \rightarrow\left(c_{0}\left|g_{-J}\right\rangle\left|2_{a}\right\rangle+c_{1}\left|g_{-J+2}\right\rangle\left|0_{a}\right\rangle\right)\left|0_{b}\right\rangle \\
& \rightarrow\left|g_{-J}\right\rangle\left(c_{0}\left|2_{a} 0_{b}\right\rangle+c_{1}\left|0_{a} 2_{b}\right\rangle\right) \equiv\left|g_{-J}\right\rangle|\psi\rangle .
\end{aligned}
$$

As the atomic state factors out in the last step, the atom can safely be discarded after completing the restoration.

Note that this procedure can be adapted to the inversion of $\hat{B}$-type jumps simply by changing the Raman $\pi$ pulse to a $3 \pi$ pulse during restoration of the state of resonator b. Also, the entire setup could be simplified by using two optical modes of opposite circular polarization in a single Fabry-Pérot cavity. The appropriate observation basis would then be photon counting with discrimination of the linear polarization of the leaking photons.
Our second example could be implemented using trapped ions [10]. Consider an ion having a $J_{g}=\frac{1}{2} \rightarrow$ $J_{e}=\frac{3}{2}$ optical transition, with the initial state

$$
|\psi\rangle=c_{0}\left|e_{-3 / 2}\right\rangle+c_{1}\left|e_{3 / 2}\right\rangle \equiv c_{0}|0\rangle_{L}+c_{1}|1\rangle_{L} .
$$

The decay channels for this initial state are ordinary spontaneous emission, with $\left|e_{-3 / 2}\right\rangle \mapsto\left|g_{-1 / 2}\right\rangle$ producing a $\sigma_{-}$-polarized photon and $\left|e_{3 / 2}\right\rangle \mapsto\left|g_{1 / 2}\right\rangle$ producing a $\sigma_{+}$-polarized photon. Although polarization-preserving imaging of the entire dipole emission pattern would be 


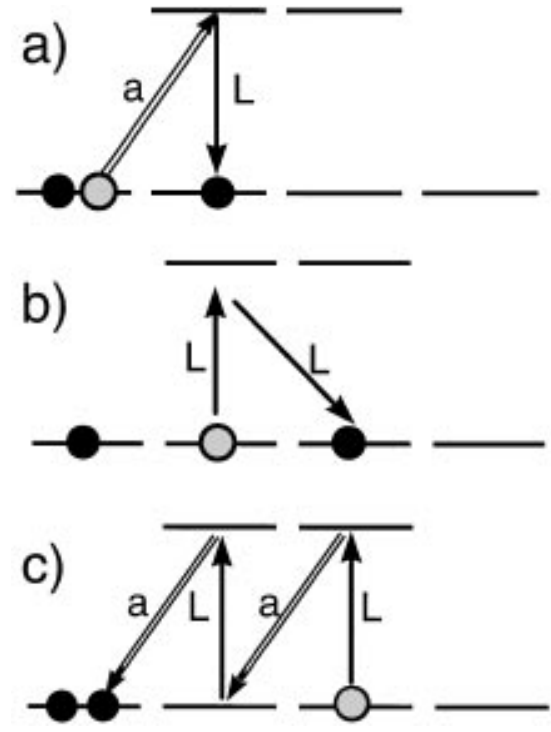

FIG. 2. Level diagram of a $J_{g}=3 / 2 \rightarrow J_{e}=1 / 2$ Zeeman atom used for photon-number doubling: (a) adiabatic state mapping via a Raman process according to Eq. (12), (b) doubling (13), and (c) mapping the Zeeman superposition back to cavity field (14) $\left(\sigma_{+}\right.$-cavity mode $a$, laser $\left.L\right)$.

experimentally difficult, let us imagine for the moment that the decay photons can be detected with perfect efficiency after the circular-polarization modes are mixed by a linearly polarizing beam splitter. The jump operators for the system are then

$$
\hat{x}, \hat{y}=\frac{1}{\sqrt{2}}\left(\left|g_{-1 / 2}\right\rangle\left\langle e_{-3 / 2}| \pm i| g_{1 / 2}\right\rangle\left\langle e_{3 / 2}\right|\right),
$$

where the operator $\hat{x}$ is associated with the detection of an $x$-polarized photon and $\hat{y}$ with the detection of a $y$-polarized photon. The associated reset operations can be achieved by simple $\pi$ pulsing on the $\pm \frac{1}{2} \rightarrow \mp \frac{3}{2}$ transitions, as long as measures are taken to avoid the unwanted but degenerate transitions $\pm \frac{1}{2} \rightarrow \mp \frac{1}{2}$. This degeneracy could be lifted by selectively shifting the $\left|e_{ \pm 1 / 2}\right\rangle$ states, for example, by applying $\pi$-polarized ac Stark fields on a transition to an auxiliary atomic level with $J_{e^{\prime}}=1 / 2$. In this scenario, the coherent restoration of superposition (16) could be verified by Ramsey-interferometry techniques. A proof-of-principle demonstration could be performed even with low photodetection efficiency by selecting the subensemble of events in which the decay photon is successfully detected [11].

The problem of storing and manipulating entangled atomic and photon states has lately attracted considerable attention within the context of recent proposals for implementing quantum computation and quantum cryptography [2]. In a quantum computer, quantum registers are defined as product states of $L$ (logical) qubits, and the general state is an entangle state of these product states. We note that state restoration by inversion of quantum jumps is also possible in such a composite system. If the subsystems (the individual qubits) are coupled to independent reservoirs, a detected decay of one of the qubits can be restored by single bit operation. Finally, we remark that the present scheme complements a recent proposal by Shor [12] on quantum error correction via redundant coding. In contrast to the Shor proposal the present scheme involves no overhead of stored and manipulated qubits, but, on the other hand, incorporates a specific quantum optical model for damping (which must be reliably known to apply to the system in question). Whereas Shor's protocol may be viewed as having quite general applicability, our scheme benefits from its context of well-established models for dissipation in concrete physical systems. In addition, we have shown recently that the methods proposed in the present paper can be extended to provide an error correction procedure for quantum gates [13].

H.M. is supported by a National Defense Science and Engineering Graduate Fellowship. This work was supported in part by the Austrian Science Foundation. Discussions with A. Barenco, T. Beth, R. Blatt, J. I. Cirac, and H. J. Kimble are acknowledged.

[1] C. W. Gardiner, Quantum Noise (Springer, Berlin, 1991).

[2] For an overview, see A. Ekert, in Proceedings of the 14th $I C A P$, edited by D. Wineland et al. (AIP Press, New York, 1995), p. 450.

[3] In a rotating frame where the optical frequencies have been eliminated.

[4] A. Barchielli and V.P. Belavkin, J. Phys. A 24, 1495 (1991), and references therein.

[5] H.J. Carmichael, in An Open Systems Approach to Quantum Optics (Springer, Berlin, 1993); J. Dalibard et al., Phys. Rev. Lett. 68, 580 (1992); C. W. Gardiner, A. S. Parkins, and P. Zoller, Phys. Rev. A 46, 4363 (1992).

[6] An operational definition of feedback in a quantum trajectory picture was first given by H. M. Wiseman and G. J. Milburn, Phys. Rev. Lett. 70, 548 (1993); H. M. Wiseman, Ph.D. thesis, University of Queensland, 1994.

[7] Equation (4) implies that an orthonormal basis (ONB) of $\mathcal{H}$ is mapped by $\hat{c}_{j}$ to an ONB of $\mathcal{H}_{s}^{(j)}$.

[8] See, for example, Cavity Quantum Electrodynamics, edited by P. R. Berman (Academic, San Diego, 1994).

[9] A. S. Parkins et al., Phys. Rev. Lett. 71, 3095 (1993).

[10] D. J. Wineland et al., Phys. Rev. A 50, 67 (1994); R. Blatt, in Proceedings of the 14th ICAP (Ref. [2]).

[11] This example illustrates clearly the relation and differences to the quantum eraser as discussed in T.J. Herzog et al., Phys. Rev. Lett. 75, 3034 (1995); P. G. Kwiat et al., Phys. Rev. A 49, 61 (1994); P. G. Kwiat et al., Phys. Rev. A 45, 7729 (1992). In the quantum eraser, interference is restored by selection of an appropriate subensemble "without path information," while in our scheme we completely restore the original state.

[12] P. W. Shor, Phys. Rev. A 52, R2493 (1995).

[13] J. I. Cirac, T. Pellizzari, and P. Zoller (unpublished); for recent experiments on quantum gates, see Q. A. Turchette et al., Phys. Rev. Lett. 75, 4710 (1995); C. Monroe et al., Phys. Rev. Lett. 75, 4714 (1995). 